\title{
Cell Line-Derived Xenograft
}

National Cancer Institute

\section{Source}

National Cancer Institute. Cell Line-Derived Xenograft. NCI Thesaurus. Code C156443.

A biospecimen derived from the culturing of a derived cell line in a non-human organism. 